\section{RANDOMTOOLS: An Apple Pascal unit useful in randomization}

\author{
BURRTON WOODRUFF \\ Butler University, Indianapolis, Indiana
}

Almost all research in the behavioral sciences requires that the ordering of the presentation of conditions be suitably randomized. The process of developing a random ordering is tedious; computer assistance is available and frequently used. Most computer languages have a built-in procedure or command for generating pseudorandom orderings. In addition, the algorithms for producing pseudorandom orderings are becoming better known (Knuth, 1969; Sander-Cederlof, 1984a), as are methods of testing the adequacy of specific implementations of algorithms (Rasmussen, 1984; Sander-Cederlof, 1984b).

Simply having a "random number" generator availa-

This work was done while the author was on a semester sabbatical leave provided by Butler University. The author's mailing address is: Department of Psychology, Butler University, 4600 Sunset Avenue, Indianapolis, IN 46208. ble on a computer still leaves large sections of program code to be written for each application program. The UCSD Pascal unit described here makes two procedure calls available to an applications program: Randomorder and Reorder. These calls greatly reduce the coding overhead for providing random orderings of sequences. Randomorder produces a linked list of pseudorandomly ordered integers ( 1 to $\mathrm{n}$ ). Randomorder also returns a heap pointer so that the memory locations used in the list can be released when no longer needed. The second procedure, Reorder, randomizes a linked list of integers provided by the calling program. When Reorder returns control to the calling program, the heap has been restored to its state at the time of the procedure call.

RANDOMTOOLS includes utility procedures making the creation, use, and maintenance of linked lists simple for those unfamiliar with this data structure. Heap management is left to the calling program. Table 1 contains a Pascal program that illustrates the use of RANDOMTOOLS by creating and then listing a random ordering of integers.

RANDOMTOOLS does not use any other UCSD units.

Table 1

Example Program Using RANDOMTOOLS

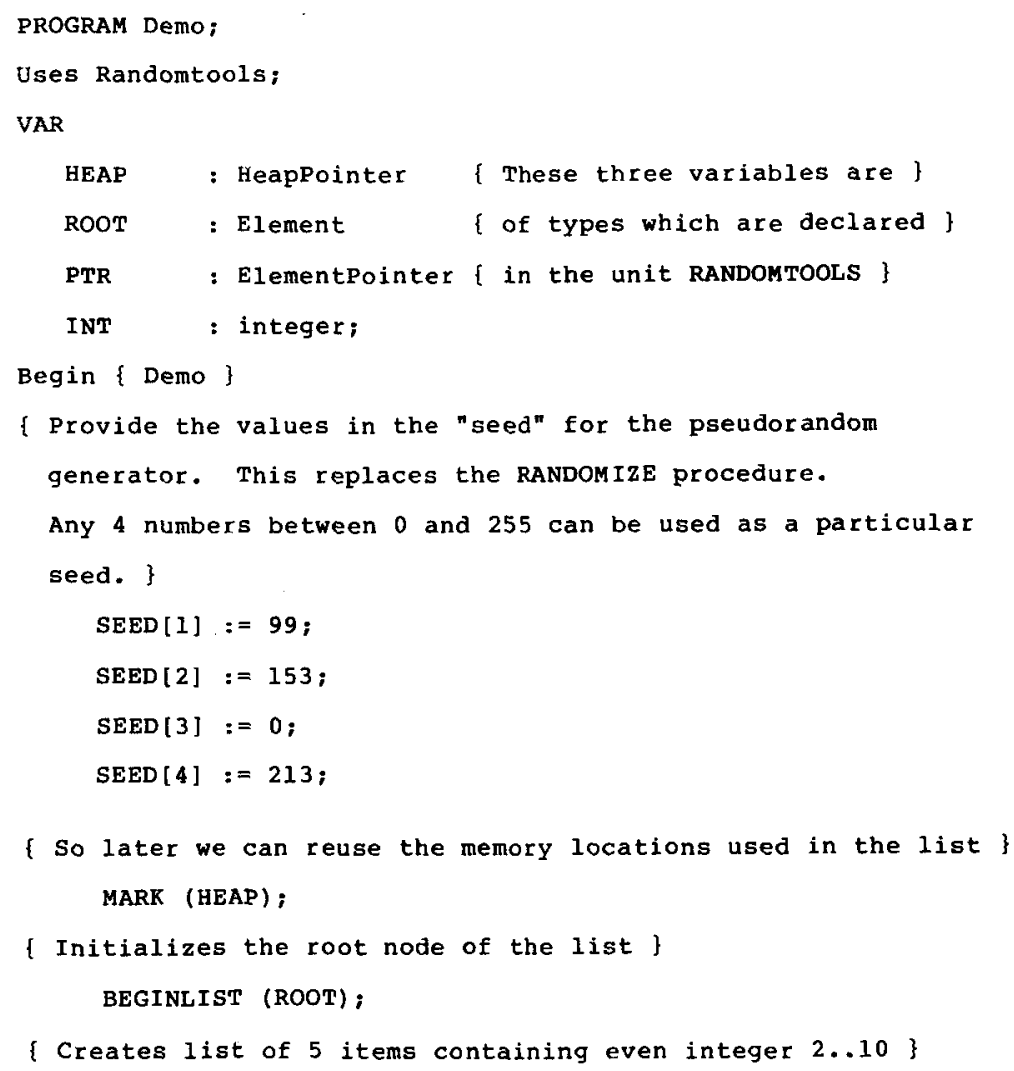




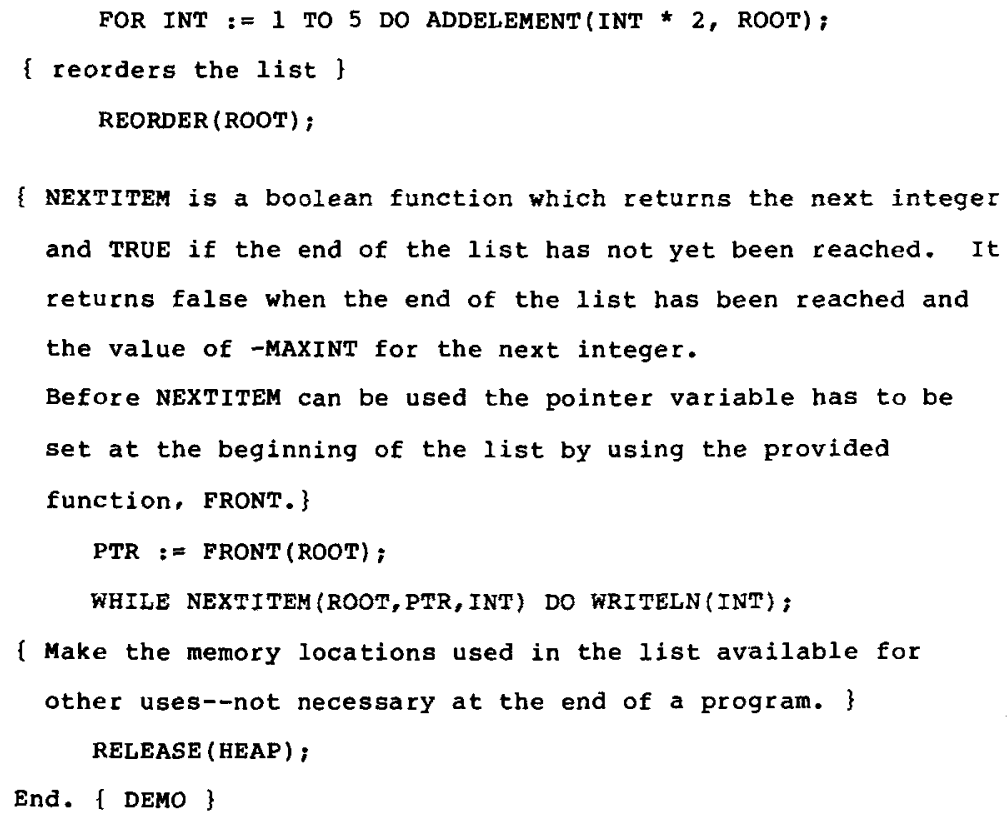

A pseudorandom generator coded in Pascal (Sands, 1984) is included. The generator is easily reseeded (see Table 1). Any other pseudorandom generator can be substituted for the one provided without changing either the basic algorithm or the data structures.

Program Requirements. The program is written in Apple Pascal v1.1 and has been used on both an Apple II + (with 16K memory card) and an Apple IIe.

Program Availability. The source code for the RANDOMTOOLS unit is available free of charge from the author. The source and object code of RANDOMTOOLS, as well as of several short demonstration programs, is available from the author on 51/4-in. diskette. Please accompany diskette requests with a $\$ 5.00$ check payable to the author.

\section{REFERENCES}

KNUTH, D. E. (1969). Semi-numerical algorithms (Vol. 2). Reading, MA: Addison-Wesley.

RASMUSSEN, J. L. (1984). A FORTRAN program for statistical evaluation of pseudorandom number generators. Behavior Research Methods, Instruments, \& Computers, 7, 63-64.

SANDS, P. A. (1984). Advanced Pascal programming techniques. Berkeley, CA: Osborn/McGraw-Hill.

SANDER-CederLof, R. (1984a). More random number generators. Apple Assembly Line, 4(9), 15-18.

SANDER-Cederlof, R. (1984b). Random numbers for Applesoft. $A p-$ ple Assembly Line, 4(8), 1-13.

(Manuscript accepted for publication February 12, 1985.) 